\title{
First documented record of Kinosternon scorpioides (Chelonia: Kinosternidae) in the state of Piauí, Northeastern Brazil
}

\author{
Etielle Barroso de Andrade
}

Grupo de Pesquisa em Biodiversidade e Biotecnologia do Centro-Norte Piauiense - BIOTECPI, Instituto Federal de Educação, Ciência e Tecnologia do Piauí - IFPI - Campus Pedro II, Rua Antonino Martins de Andrade 750, Engenho Novo 64255000, Pedro II, Piauí, Brazil. E-mail: etlandrade@hotmail.com

Andrade E.B. (2019) First documented record of Kinosternon scorpioides (Chelonia: Kinosternidae) in the state of Piauí, Northeastern Brazil. Pesquisa e Ensino em Ciências Exatas e da Natureza, 3(2): 216-223. http://dx.doi.org/10.29215/pecen.v3i2.1288

Academic editor: Samuel Cardozo Ribeiro. Received: 11 November 2019. Accepted: 16 December 2019. Published: 18 December 2019.

Primeiro registro documentado de Kinosternon scorpioides (Chelonia: Kinosternidae) no estado do Piauí, Nordeste do Brasil

Resumo: O presente trabalho fornece o primeiro registro documentado de Kinosternon scorpioides (Chelonia: Kinosternidae) no estado do Piauí e um mapa atualizado da distribuição geográfica da espécie na região Nordeste do Brasil. A espécie de ampla distribuição na América do Sul possui ainda grandes lacunas em sua distribuição geográfica. Dessa forma, o presente trabalho acrescenta dados importantes sobre a distribuição de $K$. scorpioides, especialmente na região nordeste, contribuindo para o conhecimento da biologia e conservação da espécie.

Palavras chave: Quelônio, distribuição geográfica, jurara, muçuã.

Abstract: Herein, I provide the first documented record of Kinosternon scorpioides (Chelonia: Kinosternidae) in the state of Piauí and an updated geographic distribution map of the species in Northeastern Brazil. This species of wide distribution in South America still has large gaps in its geographic distribution. Thus, I add important data on the actual distribution of $K$. scorpioides, especially in the Brazilian Northeastern region, contributing to the knowledge of the biology and conservation of this species.

Key words: Chelonian, geographic distribution, jurara, muçuã.

The turtle clade Kinosternidae is exclusive to the Americas (Iverson 1992) and consists of small to medium sized species of mud and musk turtles, widely distributed from southern Canada to northern Argentina (Turtle Taxonomy Working Group 2014). Two monophyletic subfamilies are recognized: Kinosterninae, which includes the genera Kinosternon and Sternotherus, and Staurotypinae, including the genera Staurotypus and Claudius (Iverson 1998). Kinosternon Spix, 1824 is the largest genus in the family and the most widely distributed throughout America, currently composed of 20 species (Iverson 1992; Uetz et al. 2019). Of these species, only one is found in Brazil: Kinosternon scorpioides (Linnaeus, 1766).

The scorpion mud turtle $K$. scorpioides, popularly known as muçuã or jurara, is a medium-sized chelonian (about 18 to $27 \mathrm{~cm}$ length), occur in aquatic temporary, permanent and semi-permanent habitats (Berry \& Iverson 2011), being also able to develop a semi-aquatic behavior (Pritchard \& Trebbau 1984). This species, characterized by its relatively high oval and narrow carapace, with presence of three dorsal longitudinal keels (Vinke \& Vinke 2001), has great ecological tolerance and it can be found in natural and anthropogenic water bodies (Rueda-Almonacid et al. 2007; Vogt 2008). 
Despite its extensive area of occurrence in Brazil in different biomes (Amazon, Caatinga, Cerrado, Pantanal and Coastal Zone) of the Northern, Northeastern and Midwestern regions (Iverson 1992; Cabrera \& Colantonio 1997; Costa et al. 2010; Silveira et al. 2011; Moura et al. 2014; Tomas et al. 2015), there are still large sample gaps (Silveira et al. 2011), including in the Brazilian Northeastern. The Turtle Taxonomy Working Group (2014) cites the occurrence of this species in all states of Brazilian Northeastern, however, there is no documented record of localities or vouchers (Silveira et al. 2011). Documented records of K. scorpioides occurrence in Brazilian Northeastern are found for the states of Alagoas, Bahia, Ceará, Maranhão, Paraíba, Pernambuco and Rio Grande do Norte (e.g. Cabrera \& Colantonio 1997; Berry \& Iverson 2011; Moura et al. 2014), being Pernambuco the state with the largest number of records (Moura et al. 2014) indicating a homogeneous distribution throughout the state. In other states the records are punctual or located in relatively small areas.

To date, there is no record of occurrence of the species for the states of Piauí and Sergipe. Herein, I present the first documented record of $K$. scorpioides for the state of Piauí and a geographical distribution map of this species for the Northeastern region of Brazil, filling distribution gap between the Maranhão and Ceará states.

The specimen of $K$. scorpioides (Figure 1 ) was registered in the municipality of Pedro II, located in the northern region of the state of Piauí. An adult male $(18.2 \mathrm{~cm}$ length of carapace) was accidentally found crossing an asphalted avenue near the Campus of the Federal Institute of Education, Science and Technology of Piauí - IFPI (04 $26^{\prime} 59.44^{\prime \prime}$ S, 41 $27^{\prime} 22.14^{\prime \prime}$ W) on July 4, 2019. The individual, collected to avoid its running over, was transported to the Biochemistry Laboratory of the IFPI for identification by the team of the Biodiversity and Biotechnology Research Group of Piauí North-Center - BIOTECPI. The individual was measured, photographed and released near to the Capivara River $\left(04^{\circ} 32^{\prime} 49.97^{\prime \prime} \mathrm{S}, 41^{\circ} 19^{\prime} 33.48^{\prime \prime} \mathrm{W}\right)$, located about $17.8 \mathrm{~km}$ from the collection site. The images were cataloged in the Biological Collection of the Federal Institute of Education, Science and Technology of Piauí - IFPI Campus Pedro II (CBPII 094-095).

The local where the individual was found is situated on the periphery of the municipality, an area still little urbanized that has shrub vegetation and formation of temporary small ponds during the rainy season (December to May). However, the area has been suffering constant deforestation for implementation of a residential project. The municipality of Pedro II is located in the Serra dos Matões, approximately $600 \mathrm{~m}$ above sea level, and inserted in the Environment Protection Area Serra da Ibiapaba (Brasil 1996). Pedro II is inserted in a semiarid region with transitional vegetation between the Cerrado and Caatinga (Barros et al. 2014) and has a dry and moderate climate compared to others municipalities of Piauí (Milanez \& Puppim 2009). In addition, the municipality has a large amount of perennial and temporary aquatic environments, such as the Joana weir, located about $2 \mathrm{~km}$ from the collection site, Pirapora River Waterfall, Capivara River, Urubu-Rei Waterfall, Salto Liso Waterfall, which justifies the occurrence this species in the region.

The species identification was based on the key provided by Iverson (1992) and description of Berry \& Iverson (2011), having as criteria the carapace with three longitudinal keels, plastron with two well-developed hinges, the anterior hinge located between the epiplastron and hyoplastron and the posterior hinge between hyoplastron and xiphiplastron, besides the presence of an horny spine on the prehensile tail tip, characteristics in which differentiates the species from all other Brazilian turtles species. This work corresponds the first record of $K$. scorpioides for the state of Piauí and fills a distribution gap between the Serra da Ibiapaba region, extreme west of the state of Ceará, and the northern region of the state of Maranhão (Figure 2). Localities of Northeastern Brazil with registration of the species are shown in Table 1.

Although the conservation status of $K$. scorpioides is considered as least concern (LC) (Berry \& Iverson 2011; Vogt et al. 2015), there is no accurate estimate of the species population dynamics in the wild (Carvalho Jr. et al. 2010). In addition, natural populations have been decreasing significantly in recent decades due to illegally hunt, since the 


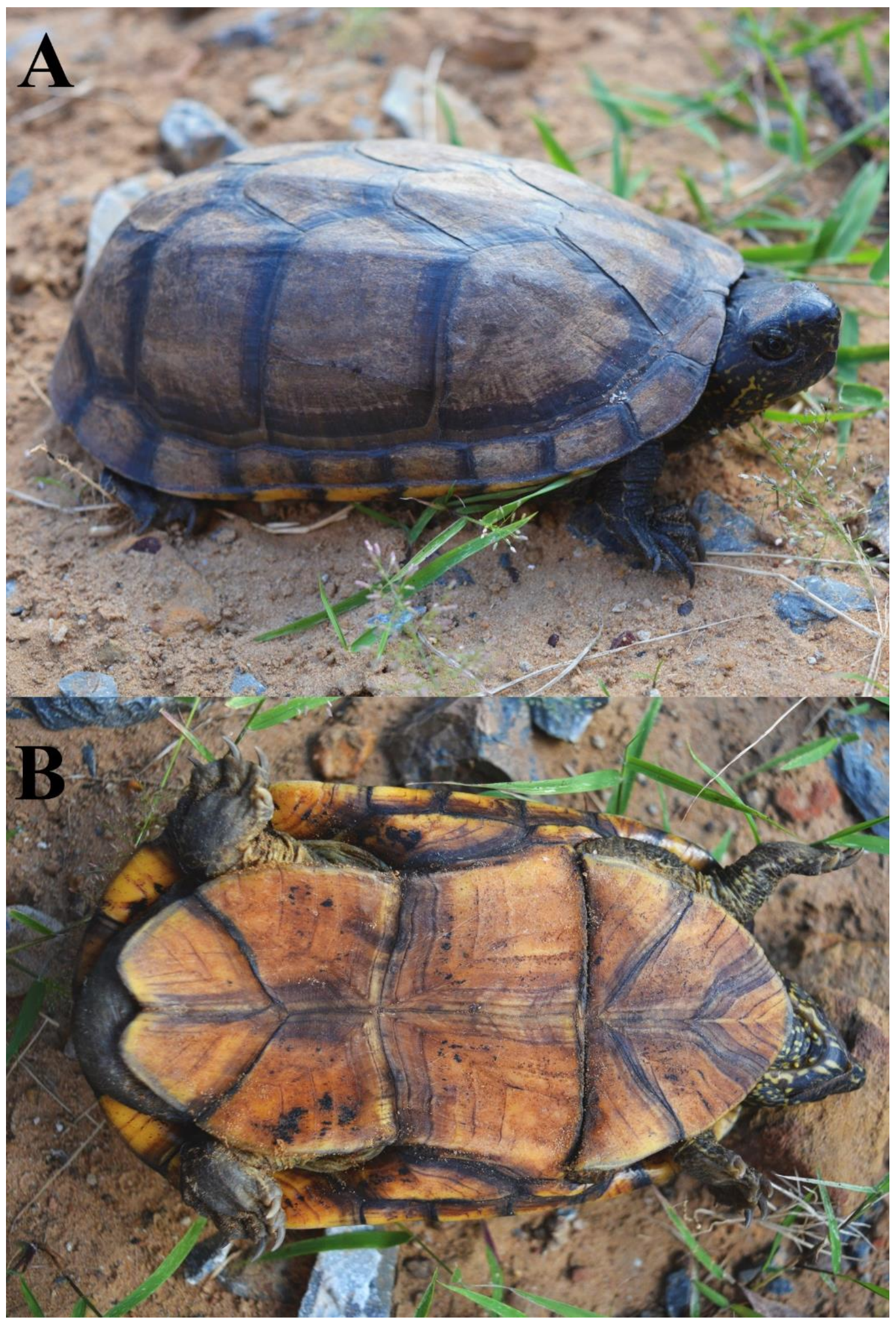

Figure 1. Adult male of Kinosternon scorpioides recorded in the municipality of Pedro II, state of Piauí, Northeastern Brazil: A. Dorsal view (CBPII 094); B. Ventral view (CBPII 095). Photo: Etielle Barroso Andrade.

species is widely appreciated in the traditional cuisine of the Brazilian Northern and Northeastern regions (Smith 1979; Vogt 2008; Alves et al. 2012) and used for medicinal purposes 
(Alves et al. 2012), for example to cure heart conditions (Leender 2001). Thus, the present work adds important data about real distribution of $K$. scorpioides, in the Northeastern Brazil, contributing to biology and conservation knowledge of the species. In addition, future studies on population dynamics in the region are fundamental to determine the ecology and actual conservation status of the species in the state of Piauí.

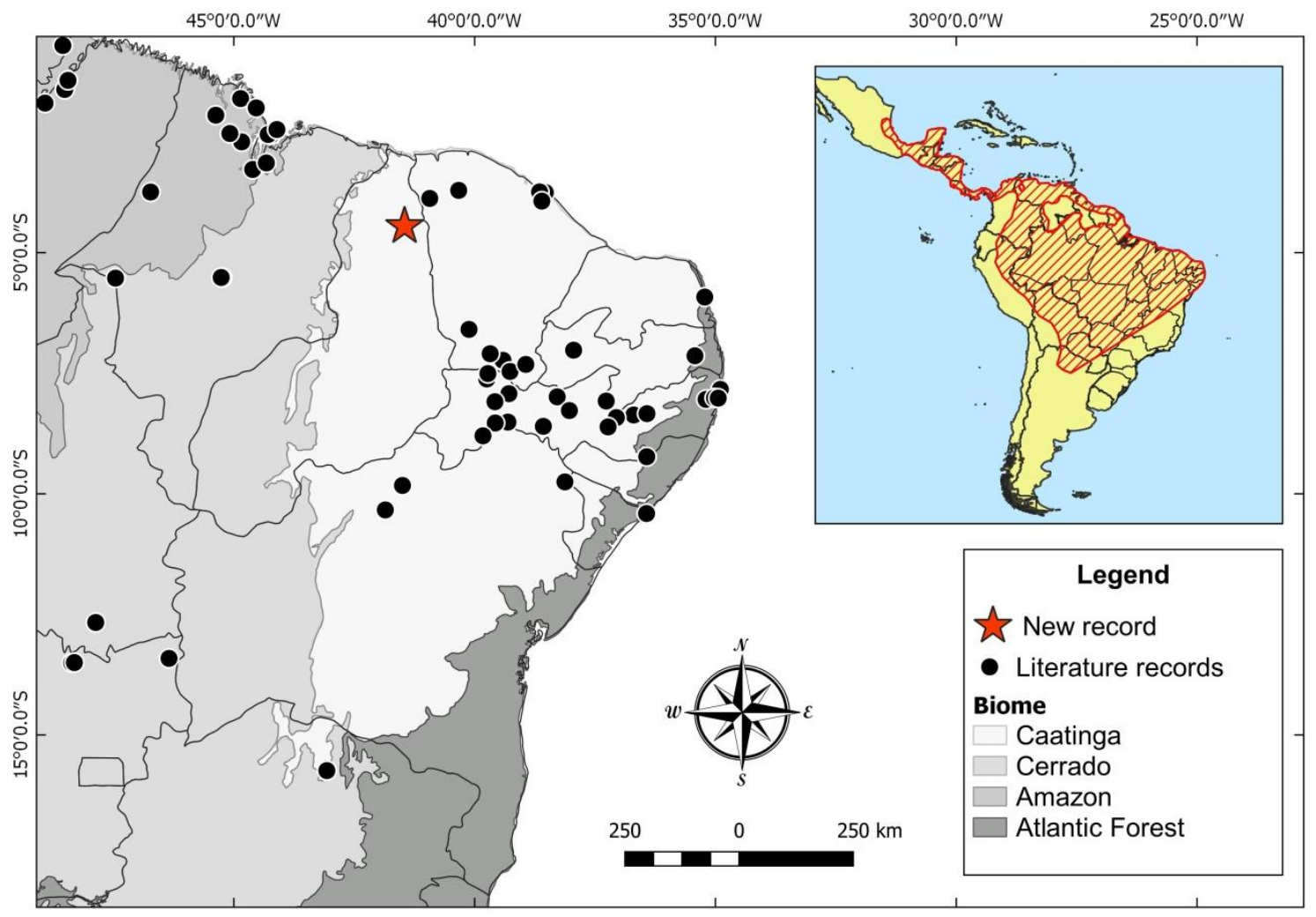

Figure 2. Geographic distribution map of Kinosternon scorpioides with new record in state of Piaui, Northeastern Brazil (red star) and literature data (black dots). The striped region represents the estimated species distribution (Turtle Taxonomy Working Group 2014).

Table 1. Localities of the Northeastern Brazil with Kinosternon scorpioides registration (- = information not available in consulted references).

\begin{tabular}{|c|c|c|c|c|}
\hline State & Municipality & Latitude (S) & Longitude (W) & Reference \\
\hline \multirow[t]{2}{*}{ Alagoas } & Piaçabuçu & $10^{\circ} 24^{\prime} 26.29^{\prime \prime}$ & $36^{\circ} 25^{\prime} 52.85^{\prime \prime}$ & Santos et al. (2008); Moura et al. (2014). \\
\hline & - & - & - & $\begin{array}{l}\text { Rodrigues (2005); Moura (2006); Moura et } \\
\text { al. (2014). }\end{array}$ \\
\hline \multirow[t]{3}{*}{ Bahia } & Santa Brígida & $09^{\circ} 44^{\prime} 00.00^{\prime \prime}$ & $38^{\circ} 08^{\prime} 00.00^{\prime \prime}$ & Santos et al. (2008); Moura et al. (2014). \\
\hline & Sento Sé & $10^{\circ} 19^{\prime} 00.00^{\prime \prime}$ & $41^{\circ} 50^{\prime} 00.00^{\prime \prime}$ & Santos et al. (2008); Moura et al. (2014). \\
\hline & - & - & - & Moura et al. (2014). \\
\hline \multirow[t]{7}{*}{ Ceará } & Aiuaba & $06^{\circ} 35^{\prime} 58.10^{\prime \prime}$ & $40^{\circ} 07^{\prime} 20.50^{\prime \prime}$ & Costa et al. (2018). \\
\hline & Caucaia & $03^{\circ} 44^{\prime} 00.46^{\prime \prime}$ & $38^{\circ} 39^{\prime} 36.54^{\prime \prime}$ & $\begin{array}{l}\text { Rocha (1948); Ribeiro et al. (2015); } \\
\text { Roberto \& Loebmann (2016). }\end{array}$ \\
\hline & Crato & - & - & $\begin{array}{l}\text { Rocha (1948); Vanzolini et al. (1980); } \\
\text { Ribeiro et al. (2015); Roberto \& Loebmann } \\
\text { (2016). }\end{array}$ \\
\hline & Fortaleza & $03^{\circ} 44^{\prime} 43.76^{\prime \prime}$ & $38^{\circ} 34^{\prime 2} 29.81^{\prime \prime}$ & $\begin{array}{l}\text { Rocha (1948); Rodrigues \& Borges-Nojosa } \\
\text { (2013); Ribeiro et al. (2015); Roberto \& } \\
\text { Loebmann (2016). }\end{array}$ \\
\hline & Milagres & $07^{\circ} 18^{\prime} 55.80^{\prime \prime}$ & $38^{\circ} 56^{\prime} 18.33^{\prime \prime}$ & $\begin{array}{l}\text { Rocha (1948); Ribeiro et al. (2015); } \\
\text { Roberto \& Loebmann (2016). }\end{array}$ \\
\hline & Nova Olinda & $07^{\circ} 05^{\prime} 37.90^{\prime \prime}$ & $39^{\circ} 40^{\prime} 42.66^{\prime \prime}$ & $\begin{array}{l}\text { Rocha (1948); Ribeiro et al. (2015); } \\
\text { Roberto \& Loebmann (2016); Pereira et al. } \\
\text { (2018). }\end{array}$ \\
\hline & Pacatuba & $03^{\circ} 55^{\prime} 46.60^{\prime \prime}$ & $38^{\circ} 36^{\prime} 20.90^{\prime \prime}$ & $\begin{array}{l}\text { Rocha (1948); Ribeiro et al. (2015); } \\
\text { Roberto \& Loebmann (2016). }\end{array}$ \\
\hline
\end{tabular}


Table 1. Continuation.

\begin{tabular}{|c|c|c|c|c|}
\hline State & Municipality & Latitude (S) & Longitude (W) & References \\
\hline & Planalto da Ibiapaba & $03^{\circ} 20^{\prime} 00.00^{\prime \prime}$ & $40^{\circ} 42^{\prime} 00.00^{\prime \prime}$ & $\begin{array}{l}\text { Rocha (1948); Loebmann \& Haddad } \\
\text { (2010); Moura et al. (2014); Ribeiro et } \\
\text { al. (2015); Roberto \& Loebmann (2016). }\end{array}$ \\
\hline & - & - & - & $\begin{array}{l}\text { Lima-Verde \& Cascon (1990); Moura et } \\
\text { al. (2014). }\end{array}$ \\
\hline \multirow[t]{12}{*}{ Maranhão } & São Bento & $02^{\circ} 42^{\prime} 05.36^{\prime \prime}$ & $44^{\circ} 50^{\prime} 13.89^{\prime \prime}$ & $\begin{array}{l}\text { Pereira et al. (2007); Barreto et al. } \\
\text { (2010); Viana et al. (2013, 2014, 2015); } \\
\text { Moura et al. (2014). }\end{array}$ \\
\hline & Anajatuba & $03^{\circ} 15^{\prime} 45.90^{\prime \prime}$ & $44^{\circ} 36^{\prime} 35.70^{\prime \prime}$ & Aragão et al. (2018). \\
\hline & Barra do Corda & $05^{\circ} 30^{\prime} 35.92^{\prime \prime}$ & $45^{\circ} 15^{\prime} 04.29^{\prime \prime}$ & $\begin{array}{l}\text { Vanzolini (1956-58); Cabrera \& } \\
\text { Colantonio (1997). }\end{array}$ \\
\hline & Cedral & $01^{\circ} 59^{\prime} 54.24 "$ & $44^{\circ} 32^{\prime} 06.38^{\prime \prime}$ & Barreto et al. (2010). \\
\hline & Ilha de Cururupu & - & - & Barreto et al. (2010). \\
\hline & Imperatriz & $05^{\circ} 31^{\prime} 32.00^{\prime \prime}$ & $47^{\circ} 28^{\prime} 37.00^{\prime \prime}$ & Viana et al. (2015). \\
\hline & Pinheiro & $02^{\circ} 31^{\prime} 31.65^{\prime \prime}$ & $45^{\circ} 04^{\prime} 58.58^{\prime \prime}$ & Barreto et al. (2010). \\
\hline & Raposa & $02^{\circ} 25^{\prime} 31.00^{\prime \prime}$ & $44^{\circ} 04^{\prime} 04.00^{\prime \prime}$ & $\begin{array}{l}\text { Barreto et al. (2009); Moura et al. } \\
\text { (2014). }\end{array}$ \\
\hline & Reserva Biológica Gurupi & $03^{\circ} 58^{\prime} 32.00^{\prime \prime}$ & $46^{\circ} 46^{\prime} 52.00^{\prime \prime}$ & Freitas et al. (2017). \\
\hline & Santa Rita & $03^{\circ} 08^{\prime} 55.97^{\prime \prime}$ & $44^{\circ} 19^{\prime} 30.19^{\prime \prime}$ & Pereira et al. (2005). \\
\hline & São Luís & $02^{\circ} 32 ' 52.92^{\prime \prime}$ & $44^{\circ} 17^{\prime} 17.77^{\prime \prime}$ & Chaves et al. (2012). \\
\hline & Turilândia & $02^{\circ} 08^{\prime} 55.48^{\prime \prime}$ & $45^{\circ} 22^{\prime} 28.23^{\prime \prime}$ & Barreto et al. (2010). \\
\hline \multirow[t]{2}{*}{ Paraíba } & Coremas & - & - & Cabrera \& Colantonio (1997). \\
\hline & Gurinhém & - & - & Cabrera \& Colantonio (1997). \\
\hline \multirow[t]{17}{*}{ Pernambuco } & Arcoverde & $08^{\circ} 25^{\prime} 06.24^{\prime \prime}$ & $37^{\circ} 03^{\prime} 30.60^{\prime \prime}$ & Freitas et al. (2019). \\
\hline & Belo Jardim & $08^{\circ} 20^{\prime} 11.40^{\prime \prime}$ & $36^{\circ} 25^{\prime} 24.60^{\prime \prime}$ & Freitas et al. (2019). \\
\hline & Betânia & $08^{\circ} 18^{\prime} 00.00^{\prime \prime}$ & $38^{\circ} 11^{\prime} 00.00^{\prime \prime}$ & Moura et al. $(2012,2014)$. \\
\hline & Cabrobó & $08^{\circ} 44^{\prime} 00.00^{\prime \prime}$ & $39^{\circ} 42^{\prime} 00.00^{\prime \prime}$ & Moura et al. $(2012,2014)$. \\
\hline & Exu & - & - & Cabrera \& Colantonio (1997). \\
\hline & Floresta & $08^{\circ} 28^{\prime} 00.00^{\prime \prime}$ & $38^{\circ} 28^{\prime} 00.00^{\prime \prime}$ & $\begin{array}{l}\text { Borges-Nojosa \& Santos (2005); Moura } \\
\text { et al. (2014). }\end{array}$ \\
\hline & Igarassu & $07^{\circ} 50^{\prime} 00.00^{\prime \prime}$ & $35^{\circ} 00^{\prime} 00.00^{\prime \prime}$ & Moura et al. $(2012,2014)$ \\
\hline & Orocó & $08^{\circ} 28^{\prime} 00.00^{\prime \prime}$ & $39^{\circ} 41^{\prime} 00.00^{\prime \prime}$ & Moura et al. (2014). \\
\hline & Parnamirim & $08^{\circ} 09^{\prime} 00.00^{\prime \prime}$ & $39^{\circ} 33^{\prime} 00.00^{\prime \prime}$ & Moura et al. (2014). \\
\hline & $\begin{array}{l}\text { Parque Nacional do } \\
\text { Catimbau }\end{array}$ & $08^{\circ} 35^{\prime} 34.57^{\prime \prime}$ & $37^{\circ} 14^{\prime} 51.24^{\prime \prime}$ & Pedrosa et al. (2015). \\
\hline & Pesqueira & $08^{\circ} 16^{\prime} 00.00^{\prime \prime}$ & $36^{\circ} 37^{\prime} 00.00^{\prime \prime}$ & Moura et al. $(2012,2014)$. \\
\hline & Recife & $08^{\circ} 00^{\prime} 52.17^{\prime \prime}$ & $34^{\circ} 56^{\prime} 38.84^{\prime \prime}$ & Melo et al. (2018). \\
\hline & Santa Maria da Boa Vista & $08^{\circ} 47^{\prime} 44.92^{\prime \prime}$ & $39^{\circ} 49^{\prime} 40.82^{\prime \prime}$ & Moura et al. (2014). \\
\hline & São Lourenço da Mata & $08^{\circ} 07^{\prime} 00.00^{\prime \prime}$ & $34^{\circ} 60^{\prime} 00.00^{\prime \prime}$ & $\begin{array}{l}\text { Santos (2009); Moura et al. (2012, } \\
\text { 2014). }\end{array}$ \\
\hline & Serra Talhada & $07^{\circ} 59^{\prime} 22.40^{\prime \prime}$ & $38^{\circ} 16^{\prime} 58.79^{\prime \prime}$ & Moura et al. (2014). \\
\hline & Serrita & $07^{\circ} 55^{\prime} 51.94 "$ & $39^{\circ} 17^{\prime} 08.28^{\prime \prime}$ & Pereira et al. (2015). \\
\hline & - & - & - & Vanzolini (1994); Santos et al. (2008). \\
\hline Piauí & Pedro II & $04^{\circ} 26^{\prime} 59.44^{\prime \prime}$ & $41^{\circ} 27^{\prime} 22.14^{\prime \prime}$ & Present study. \\
\hline $\begin{array}{l}\text { Rio Grande } \\
\text { do Norte }\end{array}$ & - & - & - & Iverson (1992); Berry \& Iverson (2011). \\
\hline
\end{tabular}

\section{Acknowledgments}

I am grateful to the librarian of the Federal Institute of Education, Science and Technology of the Pedro II Piauí-IFPI Campus Pedro II, Wirllanna Torres for the registration and transportation of the specimen to the IFPI Biochemistry Laboratory, allowing its study and contributing to the knowledge of the species in the region.

\section{References}

Alves R.R.N., Pereira Filho G.A., Vieira K.S., Souto W.M.S., Mendonça L.E.T., Montenegro P.F.G.P., Almeida W.O. \& Vieira W.L.S. (2012) A zoological catalogue of hunted reptiles in the semiarid region of Brazil. Journal of Ethnobiology and Ethnomedicine, 8: 27. http://dx.doi.org/10.1186/1746-4269-8-27 
Aragão N.R.C., Mesquita S.L., Araújo L.S.R., Macieira A.M., Chaves E.P., Chaves L.P.F., Oliveira A.S. \& Sousa A.L. (2018) Práticas de educação ambiental de jovens e adultos para a conservação de jurará (Kinosternon scorpioides) no município de Anajatuba, Baixada Maranhense. Revista Práticas em Extensão, 2(1): 08-23.

Barreto L., Lima L.C. \& Barbosa S. (2009) Observations on the ecology of Trachemys adiutrix and Kinosternon scorpioides on Curupu Island, Brazil. Herpetological Review, 40(3): 283-286.

Barreto L.N., Ribeiro L.E.S., Ribeiro A.B.N., Azevedo R.R., Tavares D.L., Abreu J.M.S. \& Cutrim N.B. (2010) Mapeamento de áreas de ocorrência e aspectos de conservação de tartarugas (Chelonia) de água doce no estado do Maranhão, Brasil. Boletim do Laboratório de Hidrobiologia, 23(1): 49.

Barros J.S., Ferreira R.V., Pedreira A.J. \& Schobbenhaus C. (2014) Geoparque Sete Cidades-Pedro II (PI): Proposta. Brasília: Serviço Geológico Brasileiro (CPRM), relatório técnico. Available in: http://www.cprm.gov.br/publique/Gestao-Territorial/Geoparques-134 (Accessed in 28/10/2019).

Berry J.F. \& Iverson J.B. (2011) Kinosternon scorpioides (Linnaeus 1766) - Scorpion Mud Turtle (p. 63.1-63.15.). In: Rhodin A.G.F., Pritchard P.C.H., Van Dijk P.P., Saumure R.A., Buhlman K.A., Iverson J.B. \& Mittermeier R.A. (Eds). Conservation Biology of Freshwater Turtles and Tortoises: A Compilation Project of the IUCN/SSC Tortoise and Freshwater Turtle Specialist Group. Chelonian Research Monographs, (5): 1-1846. http://dx.doi.org/10.3854/crm.5.063.scorpioides.v1.2011

Borges-Nojosa D.M. \& Santos E.M. (2005) Herpetofauna da área de Betânia e Floresta, Pernambuco (p. 276-289). In: Araújo F.S., Rodal M.J.N. \& Barbosa M.R.V. (Eds). Análise das Variações da Biodiversidade do Bioma Caatinga - suporte a estratégias regionais de conservação. Brasília: Ministério do Meio Ambiente. 446 p.

Brasil (1996) Decreto de 26 de novembro de 1996. Dispõe sobre a criação da Área de Proteção Ambiental Serra da Ibiapaba, nos Estados do Piauí e Ceará, e dá outras providências. Poder Executivo. Brasília-DF.

Cabrera M.R. \& Colantonio S.E. (1997) Taxonomic revision of the South American subspecies of the turtle Kinosternon scorpioides. Journal of Herpetology, 31(4): 507-513. http://dx.doi.org/10.2307/1565602

Carvalho Jr. E.A.R., Carvalho-Neto C.S. \& Paschoalini E.L. (2010) Diet of Kinosternon scorpioides in Serra dos Carajás, eastern Amazonia. Herpetological Review, 39: 283-285.

Chaves E.P., Oliveira S.C.R., Araújo L.P.F., Oliveira A.S., Miglino M.A., Abreu-Silva A.L., Melo F.A. \& Sousa A.L. (2012) Morphological aspects of the ovaries of turtle Kinosternon scorpioides raised in captivity. Pesquisa Veterinária Brasileira, 32(7): 667-671. http://dx.doi.org/10.1590/S0100-736X2012000700015

Costa H.C., Molina F.B., São-Pedro V.A. \& Feio R.N. (2010) Reptilia, Testudines, Kinosternidae, Kinosternon scorpioides scorpioides (Linnaeus, 1766): Distribution extension. Check List, 6(2): 334-335. http://dx.doi.org/10.15560/6.2.314

Costa T.B., Laranjeiras D.O., Caldas F.L.S., Santana D.O., Silva C.F., Alcântara E.P., Brito S.V., Galdino J.Y., Mesquita D.O., Faria R.G., França F.G.R., Ávila R.W. \& Garda A.A. (2018) Herpetofauna of protected areas in the Caatinga VII: Aiuaba Ecological Station (Ceará, Brazil). Herpetology Notes, 11: 929-941.

Freitas M.A., Abegg A.D., Araújo D.S., Coelho H.E.A., Azevedo W.S., Chaves M.F., Rosa C.M. \& Moura G.J.B. (2019) Herpetofauna of three "Brejos de Altitude" in the interior of the state of Pernambuco, northeastern Brazil. Herpetology Notes, 12: 591-602.

Freitas M.A., Vieira R.S., Entiauspe-Neto O.M., Sousa S.O, Farias T., Sousa A.G. \& Moura G.J.B. (2017) Herpetofauna of the Northwest Amazon forest in the state of Maranhão, Brazil, with remarks on the Gurupi Biological Reserve. Zookeys, 643: 141-155. https://doi.org/10.3897/zookeys.643.8215

Iverson J.B. (1992) A Revised Checklist with Distribution Maps of the Turtles of the World. Richmond, Indiana: Privately printed. $363 \mathrm{p}$.

Iverson J.B. (1998) Molecules, Morphology, and Mud Turtle Phylogenetics (Family Kinosternidae). Chelonian Conservation and Biology, 3(1): 113-117. 
https://doi.org/10.1016/j.ympev.2013.06.011

Leender T. (2001) A Guide to Amphibians and Reptiles of Costa Rica. Miami, Florida: Zona Tropical Publications. 304 pp.

Lima-Verde J.S. \& Cascon P. (1990) Lista Preliminar da Herpetofauna do Estado do Ceará. Caatinga, 7: 158-163.

Loebmann D. \& Haddad C.F.B. (2010) Amphibians and reptiles from a highly diverse area of the Caatinga domain: composition and conservation implications. Biota Neotropica, 10: 227-256. http://dx.doi.org/10.1590/S1676-06032010000300026

Melo Í.V., Moura G.J.B., Freitas M.A., Andrade E.V.E., Casal C., Abegg A.D. \& Kokubum M.N.C. (2018) New additions to the herpetofauna of the Dois Irmãos State Park, an urban Atlantic Rainforest fragment in northeastern Brazil. Herpetology Notes, 11: 245-254.

Milanez B. \& Puppim J.A. (2009) Ambiente, pessoas e labor: APLs além do desenvolvimento econômico na mineração de opalas em Pedro II, no Piauí. Cadernos EBAPE, 7(4): 527-546.

Moura F.B.P. (2006) A Mata Atlântica em Alagoas. Maceió: EDUFAL. 88 p.

Moura C.C.M., Guimarães E.S., Moura G.J.B., Amaral G.A. \& Silva A.C. (2012) Distribuição espaço-temporal e sucesso reprodutivo de Eretmochelys imbricata nas praias do Ipojuca, Pernambuco, Brasil. Iheringia, 102(3): 254-260. http://dx.doi.org/10.1590/S0073-47212012005000003

Moura C.C.M., Moura G.J.B., Lisboa E.B.F. \& Luz V.L.F. (2014) Distribuição geográfica e considerações ecológicas sobre a fauna de Testudines da Região Nordeste do Brasil. Sitientibus, 14: 1-20. http://dx.doi.org/10.13102/scb236

Pedrosa I.M.M.C., Costa T.B., Faria R.G., França F.G.R., Laranjeiras D.O., Oliveira T.C.S.P., Palmeira C.N.S., Torquato S., Mott T., Vieira G.H.C. \& Garda A.A. (2015) Herpetofauna of protected areas in the Caatinga III: The Catimbau National Park, Pernambuco, Brazil. Biota Neotropica, 14(4): e20140046. http://dx.doi.org/10.1590/1676-06032014004614

Pereira E.N., Teles M.J.L. \& Santos E.M. (2015) Herpetofauna em remanescente de Caatinga no Sertão de Pernambuco, Brasil. Boletim do Museu de Biologia Mello Leitão, 37(1): 29-43.

Pereira J.G., Fonseca C.C., Menin E. \& Neves M.T.D. (2005) Estudo histológico e histoquímico do esôfago do muçuã Kinosternon scorpioides Linnaeus, 1766 (Reptilia, Chelonia, Kinosternidae). Arquivos de Ciências Veterinárias e Zoologia da UNIPAR, 8(1): 3-10.

Pereira L.A., Sousa A.L., Cutrim M.V.J. \& Moreira E.G. (2007) Características ecológicas do habitat de Kinosternon scorpioides scorpioides Linnaeus, 1766 (Reptila, Chelonia, Kinosternidae) no município de São Bento - Baixada Maranhense (Maranhão, Brasil). Boletim do Laboratório de Hidrobiologia, 20: 9-14.

Pereira A.M.A., Vieira B.S., Araujo Filho J.A.A., Teixeira A.A.M., Teles D.A., Santana D.O., Lima V.F. \& Almeida W.O. (2018) Diet and helminth parasites of freshwater turtles Mesoclemmys tuberculata, Phrynops geoffroanus (Pleurodira: Chelidae) and Kinosternon scorpioides (Criptodyra: Kinosternidae) in a semiarid region, Northeast of Brazil. Acta Herpetologica, 13(1): 21-32.

Pritchard P.C. \& Trebbau P. (1984) The turtles of Venezuela. Ithaca: Society the Study of Amphibians and Reptiles. 403 p.

Ribeiro S.C., Roberto I.J., Oliveira H.F., Oliveira R.H., da Silva M.C., Almeida W.O. \& Ávila R.W. (2015) Herpetofauna da Chapada do Araripe: composição, distribuição e conservação (p. 235272). In: Albuquerque U.P. \& Meiado M.V. (Eds). Sociobiodiversidade na Chapada do Araripe. Recife: Nupeea. 536 p.

Roberto I.J. \& Loebmann D. (2016) Composition, distribution patterns, and conservation priority areas for the herpetofauna of the state of Ceará, northeastern Brazil. Salamandra, 52: 134152.

Rocha F.D. (1948) Subsídio para o estudo da fauna cearense (Catálogo das espécies animais por mim coligidas e notadas). Revista do Instituto do Ceará, 62: 102-138.

Rodrigues M.T. (2005) Conservação dos répteis brasileiros: os desafios de um país megadiverso. Megadiversidade, 1(1): 88-94. 
Rodrigues J.F.M. \& Borges-Nojosa D.M. (2013) Does Kinosternon scorpioides (Linnaeus, 1766) (Testudines: Kinosternidae) prefer to reproduce in clean water? Herpetology Notes, 6: 519521.

Rueda-Almonacid J.V., Carr J.L., Mittermeier R.A., Rodriguez-Marecha J.V., Mast R.B., Vogt R.C., Rhodin A.G., Ossa-Velásquez J., Rueda J.N. \& Mittermeier C.G. (2007) Las tortugas e los cocodrilianos de los países andinos del trópico. Serie de guias tropicales de campo $\mathrm{N}^{\circ} 6$. Bogotá: Conservación Internacional Editorial Panamericana, Formas e Impresos. 537 p.

Santos E.M. (2009) Notas sobre predação de anuros em uma poça temporária no nordeste do Brasil. Boletim do Museu de Biologia Mello Leitão, 25: 77-82.

Santos F.J.M., Pena A.P. \& Luz V.L.F. (2008) Considerações biogeográficas sobre a herpetofauna do submédio e da foz do rio São Francisco, Brasil. Estudos, 35(1/2): 59-78.

Silveira A.L., Balestra R.A.M., Ferreira D.I., Luz V.L.F., Pena A.P. \& Coutinho M.E. (2011) Primeiro registro documentado de Kinosternon scorpioides scorpioides (Reptilia, Testudines, Chelidae) no Estado de Minas Gerais, Sudeste do Brasil. Revista Brasileira de Zoociências, 13: 249-251.

Smith N.J.H. (1979) Aquatic turtles of Amazonia: an endangered resource. Biological Conservation, 16: 1165-1176.

Tomas W.M., Chiaravalotti R.M., Camilo A.R. \& Freitas G.O. (2015) Kinosternon scorpioides scorpioides Linnaeus, 1766: range extension and first records in the upper Paraguay River basin and Mato Grosso do Sul, Brazil. Check List, 11(3): 1631.

http://dx.doi.org/10.15560/11.3.1631

Turtle Taxonomy Working Group (2014) Turtles of the world. $7^{\circ}$ edition: Annotated checklist of taxonomy, synonymy, distribution with maps, and conservation status. Chelonian Research Monographs, 5: 329-479.

Uetz P., Freed P. \& Hošek J. (2019) The Reptile Database. Available in: http://www.reptiledatabase.org (Accessed 29/10/2019).

Vanzolini P.E. (1956-58) Notas sobre a zoologia dos índios Canela. Revista do Museu Paulista, 10: 155-171.

Vanzolini P.E. (1994) On the distribution of certain South American turtles (Testudines: Testudinidae \& Chelidae). Smithsonian Herpetological Information Service, 97: 1-10.

Vanzolini P.E., Ramos-Costa A.M.M. \& Vitt L.J. (1980) Répteis das caatingas. Rio de Janeiro: Academia Brasileira de Ciências. 161 p.

Viana D.C., Santos A.C. \& Antunes R.L.S. (2015) New record of Kinosternon scorpioides in Brazil increases its geo distribution - Case report. Revista Brasileira de Medicina Veterinária, 37(4): 386-388.

Viana D.C., Rui L.A., Miglino M.A., Araujo L.P.F., Oliveira A.S. \& Sousa A.L. (2013) Morphological study of epididymides in the scorpion mud turtle in natural habitat (Kinosternon scorpioides - Linnaeus, 1976). Biotemas, 26(2): 153-162.

Viana D.C., Rui L.A., Santos A.C., Miglino M.A., Assis Neto A.C., Araujo L.P., Oliveira A.S. \& Sousa A.L. (2014) Seasonal morphological variation of the vas deferens of scorpion mud turtle (Kinosternon scorpioides). Biota Neotropica, 14(3): 1-5. http://dx.doi.org/10.1590/167606032014006413

Vinke T. \& Vinke S. (2001) The turtle and tortoise fauna of the central Chaco of Paraguay. Radiata, 10(3): 3-19.

Vogt R.C. (2008) Amazon turtles. Lima, Peru: Grafica Biblio. 104 p.

Vogt R.C., Fagundes C.K., Bataus Y.S.L., Balestra R.A.M., Batista F.R.W., Uhlig V.M., Silveira A.L., Bager A., Batistella A.M., Souza F.L., Drummond G.M., Reis I.J., Bernhard R., Mendonça S.H.S.T. \& Luz V.L.F. (2015) Avaliação do Risco de Extinção de Kinosternon scorpioides (Linnaeus, 1766) no Brasil. Processo de avaliação do risco de extinção da fauna brasileira. ICMBio. Available in: http://www.icmbio.gov.br/portal/biodiversidade/fauna-brasileira/estadode-conservacao/7407-repteis-kinosternon-scorpioides-mucua.html (Accessed 29/10/2019). 